\title{
Adaptación del Cuestionario de Fuentes de Confianza Deportiva (SSCQ) al contexto español
}

\section{Adaptation of Sources of the Sport Confidence Questionnaire (SSCQ) to the Spanish context}

\author{
Constantino Arce, Javier Garrido, Elena Andrade, Julio Torrado, \\ Cristina de Francisco, Iria Arce
}

Universidad de Santiago de Compostela

Disponible online 30 de abril de 2010

\begin{abstract}
Este estudio constituye un primer paso en la adaptación del Cuestionario de Fuentes de Confianza en el Deporte (Sources of Sport Confidence Questionnaire, SSCQ) al contexto español y, en particular, al deporte del fútbol. En el proceso se siguieron tres fases, describiéndose aquí la fase 3 en la que se sometió a análisis factorial confirmatorio la versión traducida del cuestionario original al español, con un total de 43 ítems agrupados en 9 factores representativos de 9 fuentes de confianza habitualmente utilizadas por los deportistas. Se utilizó una muestra de 402 futbolistas juveniles federados. Análisis confirmatorios sucesivos lograron replicar una versión española del SSCQ con 34 ítems agrupados en 8 factores o fuentes de confianza. Los índices de ajuste global del modelo fueron satisfactorios y las cargas factoriales todas ellas significativas. Además, de las buenas propiedades psicométricas de la versión española del cuestionario también se ha comprobado que su aplicación es sencilla y fácilmente comprensible para los deportistas. De cara al fututo, sería conveniente desarrollar versiones más generales del cuestionario que puedan ser aplicadas en cualquier deporte. También se debería estudiar la invarianza de la estructura de las fuentes de confianza en función del género y la edad de los deportistas.
\end{abstract}

Palabras clave: Confianza deportiva, fuentes de confianza, adaptación cultural, cuestionario

This study constitutes the first step in adapting the Sources of Sport Confidence Questionnaire (SSCQ) to the Spanish context and, in particular, to the sport of football. Three phases were adhered to in the process; the current paper describes Phase 3, in which the translated Spanish version of the original questionnaire-with a total of 42 items grouped into 9 factors representing the 9 sources of confidence habitually used by athletes-was subjected to a confirmatory factor analysis. A sample comprising 402 young federated footballers was employed. Successive confirmatory analysis successfully replicated a Spanish version of the SSCQ, with 34 items grouped into 8 factors or sources of confidence. The model's global fit indices were satisfactory and all factorial loads significant. In addition, owing to the excellent psychometric properties of the Spanish version of the questionnaire, it was also verified that it was simple to apply and easily understandable for the athletes. With regard to future studies, it would be expedient to develop less specific versions of the questionnaire which can be applied to any sport. The invariance in the structure of the sources of confidence depending on the gender and age of athletes will also need to be addressed.

Keywords: Sport confidence, sources of confidence, cultural adaptation, questionnaire

\footnotetext{
La presente investigación ha sido realizada con la ayuda de un proyecto de investigación financiado por la Consellería de Economía e Industria de la Xunta de Galicia (PGIDIT06PXIB211187PR).

Correspondencia: Constantino Arce. Universidad de Santiago de Compostela. 15782. Santiago de Compostela. España. E-mail: constantivo.arce@usc.es
} 
El objetivo del presente trabajo es realizar una traducción al español del Sources of Sport Confidence Questionnaire (SSCQ) de Vealey, Hayashi, Garner-Holman y Giacobbi (1998), e iniciar la adaptación de dicho cuestionario tanto al contexto español, como al deporte del fútbol específicamente.

El Sources of Sport Confidence Questionnaire (Vealey et al., 1998) fue diseñado tras llevar a cabo una revisión del modelo original de confianza en el deporte de Vealey (1986). En este modelo revisado se conceptualizaron diversas fuentes de confianza en el deporte, y utilizando entrevistas, cuestionarios de preguntas abiertas, y una revisión de la literatura relevante se desarrolló el SSCQ para identificar las fuentes de confianza más importantes.

El estudio y desarrollo del instrumento original se realizó en cuatro fases. En las tres primeras fases se desarrollaron las bases conceptuales preliminares para las fuentes de confianza en el deporte y se encontró la primera evidencia psicométrica que apoyaba la estructura factorial, la fiabilidad y la validez del SSCQ. En la fase cuatro se llevó a cabo un análisis factorial confirmatorio para demostrar la estructura multidimensional del cuestionario.

Los ítems fueron elaborados para representar nueve fuentes de confianza en el deporte: maestría, demostración de habilidad, preparación física y mental, autopresentación física, apoyo social, liderazgo del entrenador, experiencia vicaria, comodidad del entorno y favorecimiento situacional. El SSCQ original ha resultado ser un cuestionario válido para medir las fuentes de confianza en el deporte. Y se han encontrado valores de alfa de Cronbach superiores a 0.70 , en todas sus subescalas, siendo en algún caso menor la consistencia interna debido al menor número de ítems en esas subescalas (Vealey el al., 1998).

\section{Método}

\section{Participantes}

La muestra estuvo formada en la primera fase por 402 sujetos, todos ellos jugadores de fútbol de categoría juvenil, federados en la Comunidad Autónoma de Galicia. El rango de edad de los participantes iba desde los 14 hasta los 19 años, siendo la media de edad de 17.06 y la desviación típica de 0.98 .

\section{Instrumento}

El instrumento de medida utilizado fue una versión traducida y adaptada al español del Sources of Sport Confidence Questionnaire (Vealey et al., 1998), que fue elaborada teniendo en cuenta recomendaciones de la literatura sobre adaptación de test y escalas (Hambleton, 1996; Muñiz y Hambleton, 2000). El cuestionario está formado por 43 ítems referidos a las 9 fuentes de confianza en el deporte, mencionadas anteriormente, que mide el SSCQ, formando las 9 subescalas o dimensiones del cuestionario. La escala de respuesta fue tipo Likert con 7 alternativas de respuesta (1-7), siendo 1 nada importante y 7 muy importante.

\section{Procedimiento}

El cuestionario fue aplicado mediante un procedimiento estandarizado. Se contactaba con los entrenadores de los equipos para acudir a un entrenamiento y realizar el ejercicio en los minutos previos al entreno. La administración se llevó a cabo de forma colectiva en los vestuarios del club. Los datos eran recogidos por el investigador y psicólogos deportivos. Los sujetos recibían siempre las mismas instrucciones a la vez que se les explicaba el propósito de la investigación. Se les indicaba que pensasen en un momento o situación en la que se hubiesen sentido muy confiados cuando competían, y que pensasen sobre qué tipo de cosas le hacían sentirse confiado en esas situaciones. Después debían responder a cada ítem indicando la importancia que esa fuente, representada por el ítem en particular, tenía para que él o ella se sintiera confiado compitiendo en su deporte.

\section{Resultados}

En primer lugar se sometió a análisis factorial confirmatorio con el programa LISREL 8.80 el modelo original de Vealey et al. (1998) formado por 43 ítems agrupados en nueve factores, correspondientes con las nueve fuentes de confianza en el deporte identificadas en dicho modelo. Se especificaron las relaciones entre cada ítem y su factor correspondiente y se permitieron relaciones entre factores. El método de estimación empleado fue el de máxima verosimilitud. Para la evaluación del ajuste se siguieron los criterios de Schermelleh-Engel, Moosbrugger y Müller (2003). Se tomó la decisión de eliminar el ítem "Siento que todo va bien" en base a los siguientes criterios: presentó la menor carga factorial de la totalidad de los ítems, mostró los índices de modificación más elevados y los residuales estandarizados de mayor valor. Este ítem pertenecía al factor denominado "Favorecimiento situacional", formado originalmente por tres ítems que evaluaban esta fuente de confianza. Con la eliminación de este ítem el factor quedaba representado por sólo dos ítems, lo que sugería prescindir de este factor, dado que no se podía asumir que estuviese medido por solamente dos indicadores.

El nuevo modelo así reducido se sometió de nuevo a análisis factorial confirmatorio. Sustantivamente, los 40 ítems reflejaban ocho fuentes de confianza teóricas. Se observaron y tuvieron en cuenta los índices de ajuste individual, eliminando sucesivamente aquellos ítems que presentaban valores bajos en su carga factorial que, a su vez, mostraban índices de modificación altos debido a su relación con otros factores, y presentaban también valores altos en sus residuos estandarizados en relación a ítems de otras dimensiones. 
Se eliminaron secuencialmente los siguientes ítems: "Gano", "Consigo ganar y clasificarme", pertenecientes al factor Demostración de habilidad; "Sigo ciertos rituales", perteneciente al factor Comodidad del entorno; "Recibo apoyo de los entrenadores", perteneciente al factor Apoyo social; "Observo como los demás están a mi nivel”, perteneciente al factor Experiencia vicaria; y "Recibo ánimos de mi familia", perteneciente al factor Apoyo social. Tras las reespecificaciones el nuevo modelo quedó formado por 34 ítems agrupados en ocho factores latentes. Obteniéndose un ajuste satisfactorio. Los índices de ajuste global se presentan en la Tabla 1. En la Tabla 2 se muestran los ítems del modelo definitivo con las estimaciones de las cargas factoriales y su correspondiente valor $t$.

Tabla 1. Índices de ajuste del modelo.

\begin{tabular}{lc}
\hline \multicolumn{1}{c}{ Índice } & Valor \\
\hline$\chi^{2} /$ df: Chi-cuadrado / Grados de Libertad & 2.24 \\
RMSEA: Error cuadrático medio de aproximación & 0.056 \\
RMR: Raíz media cuadrática residual & 0.057 \\
CFI: Índice de ajuste comparativo & 0.95 \\
NNFI: Índice de ajuste no normado & 0.95 \\
\hline
\end{tabular}

También se evaluaron las correlaciones (Tabla 3) entre los factores estimadas por el programa estadístico en el análisis factorial confirmatorio. Todas las correlaciones fueron significativas excepto las relaciones entre los factores Liderazgo del entrenador y Demostración de habilidad y entre los factores Experiencia vicaria y Demostración de habilidad. Las correlaciones más altas se dieron entre los factores Comodidad del entorno y Apoyo social (0.72), Comodidad del entorno y Preparación física y mental (0.64) y Experiencia vicaria y Liderazgo del entrenador (0.63).

Por otro lado, las correlaciones de menor valor fueron las estimadas entre Apoyo social y Autopresentación fisica (0.21), y entre Autopresentación física y Demostración de habilidad (0.25). En base a estos datos y desde el punto de vista teórico no se encontraron evidencias que aconsejaran un cambio en el número de factores del modelo.

Tabla 3. Correlaciones entre factores.

\begin{tabular}{lcccccccc}
\hline Factor & M & DH & PFM & APF & AS & LE & CE & EV \\
\hline M & 1 & & & & & & & \\
DH & 0.62 & 1 & & & & & & \\
PFM & 0.54 & 0.36 & 1 & & & & & \\
APF & 0.31 & 0.25 & 0.30 & 1 & & & & \\
AS & 0.47 & 0.31 & 0.55 & 0.21 & 1 & & & \\
LE & 0.30 & $0.07 *$ & 0.56 & 0.19 & 0.55 & 1 & & \\
CE & 0.44 & 0.34 & 0.64 & 0.23 & 0.72 & 0.45 & 1 & \\
EV & 0.37 & $0.01^{*}$ & 0.44 & 0.38 & 0.43 & 0.63 & 0.43 & 1 \\
\hline
\end{tabular}

M: Maestría; DH: Demostración de Habilidad; PFM: Preparación Física y Mental; APF: Autopresentación Física; AS: Apoyo Social; LE: Liderazgo del Entrenador; CE: Comodidad del Entorno; EV: Experiencia Vicaria
Tabla 2. Estimaciones de las cargas factoriales y valor $t$.

\begin{tabular}{|c|c|c|c|}
\hline Factor & Ítem & $\begin{array}{l}\text { Carga } \\
\text { Factorial } \\
\text { Estimada }\end{array}$ & Valor $\mathbf{t}$ \\
\hline \multirow{5}{*}{ Maestría } & Domino nuevas habilidades & 0.69 & 12.82 \\
\hline & $\begin{array}{l}\text { Mejoro en alguna acción } \\
\text { técnica }\end{array}$ & 0.69 & 11.92 \\
\hline & $\begin{array}{l}\text { Mejoro mis habilidades } \\
\text { técnicas }\end{array}$ & 0.71 & 11.68 \\
\hline & $\begin{array}{l}\text { Aumento el número de } \\
\text { acciones técnicas que puedo } \\
\text { realizar }\end{array}$ & 0.68 & 11.95 \\
\hline & $\begin{array}{l}\text { Desarrollo nuevas habilida- } \\
\text { des técnicas y mejoro }\end{array}$ & 0.74 & 11.19 \\
\hline \multirow{6}{*}{$\begin{array}{l}\text { Preparación } \\
\text { Física y Mental }\end{array}$} & $\begin{array}{l}\text { Mantengo mi concentración } \\
\text { en el juego }\end{array}$ & 0.57 & 12.82 \\
\hline & Consigo mentalizarme & 0.53 & 13.05 \\
\hline & $\begin{array}{l}\text { Sé que estoy mentalmente } \\
\text { preparado }\end{array}$ & 0.66 & 11.94 \\
\hline & $\begin{array}{l}\text { Me mantengo centrado en lo } \\
\text { que tengo que hacer }\end{array}$ & 0.65 & 12.12 \\
\hline & $\begin{array}{l}\text { Me preparo física y mental- } \\
\text { mente }\end{array}$ & 0.76 & 10.38 \\
\hline & $\begin{array}{l}\text { Creo que puedo esforzarme } \\
\text { al máximo }\end{array}$ & 0.53 & 13.04 \\
\hline \multirow{4}{*}{$\begin{array}{l}\text { Demostración } \\
\text { de Habilidad }\end{array}$} & $\begin{array}{l}\text { Demuestro que soy mejor } \\
\text { que los otros }\end{array}$ & 0.67 & 12.28 \\
\hline & $\begin{array}{l}\text { Sé que técnicamente soy } \\
\text { mejor que mis rivales }\end{array}$ & 0.74 & 11.32 \\
\hline & $\begin{array}{l}\text { Demuestro que soy mejor } \\
\text { que el rival }\end{array}$ & 0.82 & 9.15 \\
\hline & $\begin{array}{l}\text { Demuestro que soy uno de } \\
\text { los mejores }\end{array}$ & 0.78 & 10.55 \\
\hline \multirow{4}{*}{ Apoyo Social } & $\begin{array}{l}\text { Veo que mis compañeros me } \\
\text { animan }\end{array}$ & 0.62 & 11.63 \\
\hline & $\begin{array}{l}\text { Sé que cuento con el apoyo } \\
\text { de los demás }\end{array}$ & 0.68 & 10.45 \\
\hline & Sé que los demás creen en mi & 0.66 & 10.89 \\
\hline & El público me anima & 0.38 & 13.45 \\
\hline \multirow{5}{*}{$\begin{array}{l}\text { Liderazgo del } \\
\text { Entrenador }\end{array}$} & $\begin{array}{l}\text { Creo en las capacidades de } \\
\text { mi entrenador }\end{array}$ & 0.66 & 12.87 \\
\hline & $\begin{array}{l}\text { Sé que mi entrenador va a } \\
\text { tomar decisiones acertadas }\end{array}$ & 0.79 & 11.31 \\
\hline & $\begin{array}{l}\text { Sé que el entrenador es un } \\
\text { buen líder }\end{array}$ & 0.82 & 10.76 \\
\hline & $\begin{array}{l}\text { Creo en las decisiones de mi } \\
\text { entrenador }\end{array}$ & 0.81 & 10.85 \\
\hline & $\begin{array}{l}\text { Siento que mi entrenador } \\
\text { actúa como un líder }\end{array}$ & 0.76 & 11.85 \\
\hline \multirow{4}{*}{$\begin{array}{l}\text { Experiencia } \\
\text { Vicaria }\end{array}$} & Veo jugar un buen partido & 0.53 & 12.93 \\
\hline & Veo a otro futbolista jugar & 0.61 & 12.30 \\
\hline & $\begin{array}{l}\text { Observo como un compañero } \\
\text { juega bien }\end{array}$ & 0.74 & 10.22 \\
\hline & Veo a un amigo jugar bien & 0.78 & 9.27 \\
\hline \multirow{3}{*}{$\begin{array}{l}\text { Autopresenta- } \\
\text { ción Física }\end{array}$} & Me siento bien con mi peso & 0.62 & 12.02 \\
\hline & $\begin{array}{l}\text { Me siento bien con mi } \\
\text { aspecto }\end{array}$ & 0.74 & 9.23 \\
\hline & $\begin{array}{l}\text { Me siento a gusto con mi } \\
\text { cuerpo }\end{array}$ & 0.85 & 5.59 \\
\hline \multirow{3}{*}{$\begin{array}{l}\text { Comodidad del } \\
\text { Entorno }\end{array}$} & $\begin{array}{l}\text { Juego en un ambiente que } \\
\text { me gusta }\end{array}$ & 0.62 & 11.78 \\
\hline & $\begin{array}{l}\text { Me siento cómodo en el } \\
\text { campo }\end{array}$ & 0.60 & 11.98 \\
\hline & $\begin{array}{l}\text { Me gusta el ambiente en el } \\
\text { que juego }\end{array}$ & 0.79 & 11.95 \\
\hline
\end{tabular}


Con este trabajo se ha dado el primer paso para la adaptación al contexto español, y particularmente al deporte del fútbol, del SSCQ. Durante el proceso de traducción se puso especial énfasis en conseguir la mayor equivalencia entre los ítems en inglés y su forma en español. El funcionamiento del cuestionario a este nivel se consideró muy satisfactorio, puesto que fue fácil de aplicar y de comprender por parte de los sujetos. Por lo tanto, este estudio ha constituido un primer paso en la adaptación de SSCQ al contexto español. Los resultados mostraron que los datos reproducen 8 de las 9 dimensiones que inicialmente se consideraron en el modelo, y que fueron etiquetadas acorde con el planteamiento teórico. Basándose en los resultados e índices de ajuste del análisis factorial confirmatorio, se eliminó la dimensión denominada "Favorecimiento situacional" debido al mal funcionamiento de sus ítems en nuestra población y secuencialmente también se procedió a eliminar una serie de ítems que presentan pobres índices de ajuste individual. Con este estudio se ha aportado evidencia del funcionamiento del SSCQ en nuestro contexto, obteniendo una solución que se corresponde esencialmente con la estructura original. Sin embargo se han encontrado limitaciones en algunos de los ítems del cuestionario original, y el factor "Favorecimiento situacional" que parece no ser identificado como una fuente de confianza relevante en nuestro contexto.

Con respecto a investigaciones futuras pueden tenerse en cuenta las siguientes consideraciones:

a) En el presente estudio hemos trabajado con una muestra específica de futbolistas y con un rango de edad restringido, por lo que la valoración e importancia percibida de las fuentes de confianza puede ser diferente en otros deportes y edades.

b) Intentos previos como el de Wilson, Sullivan, Myers y Feltz (2002) no han conseguido replicar la estructura factorial original del SSCQ en diferentes poblaciones.

c) Queda por determinar la invarianza de la estructura de las fuentes de confianza en función del género así como la existencia de posibles diferencias en la valoración e importancia de las fuentes de confianza entre deportistas masculinos y femeninos.
Hambleton, R.K. (1996). Adaptación de test para su uso en diferentes idiomas y culturas: fuentes de error, posibles soluciones y directrices prácticas. En J. Muñiz (Coord.), Psicometría (pp. 207-238). Madrid: Universitas.

Muñiz, J., y Hamblenton, R. (2000). Adaptación de los test de unas culturas a otras. Metodología de las ciencias del comportamiento, 2, 129-149.

Schermelleh-Engel, K., Moosbrugger, H. y Müller, H. (2003). Evaluating the fit of structural equation models: Test of significance and descriptive goodness-of-fit measures. Methods of Psychological Research - Online, 8, 23-74.

Vealey, R.S. (1986). Conceptualization of sport-confidence and competitive orientation: Preliminary investigation and instrument development. Journal of Sport Psychology, 8, 221-246.

Vealey, R.S., Hayashi, S.W., Garner-Holman, G., y Giacobbi, P. (1998).Source of Sport-confidence: Conceptualization and instrument development. Journal of Sport and Exercise Psychology, 20, 54-80.

Wilson, R.C., Sullivan, P.J., Myers, N.D., y Feltz, D.L. (2004). Sources of Sport Confidence of Master Athletes. Journal of Sport and Exercise Psychology, 26, 369-384.

Fecha de recepción: 25 de septiembre de 2009 Fecha de aceptación: 26 de enero de 2009 